\title{
Towards Precision Determination of uPDFs
}

\author{
Magnus Hansson ${ }^{1}$ and Hannes Jung ${ }^{2}$ \\ 1- Lund University \\ 2- DESY, FRG
}

\begin{abstract}
The unintegrated Parton Density Function of the gluon is obtained from a fit to dijet production in DIS as measured at HERA. Reasonable descriptions of the measurements are obtained, and a first attempt to constrain the intrinsic transverse momentum distribution at small $k_{\perp}$ is presented [1].
\end{abstract}

\section{Introduction}

Unintegrated parton density functions (uPDFs) are best suited to study details of the hadronic final state in high energy ep and also in $p p$ collisions (for a review see [2-8]). In general, the production cross section for jets, heavy quarks or gauge bosons can be written as a convolution of the uPDF $\mathcal{A}\left(x, k_{\perp}^{2}, \bar{q}\right)$ with the partonic off-shell cross section $\hat{\sigma}\left(x_{i}, k_{\perp}^{2}\right)$, with $x_{i}, k_{\perp}$ being the longitudinal momentum fraction and the transverse momentum of the interacting parton $i$ and $\bar{q}$ being the factorization scale. For example the cross section for $e p \rightarrow$ jets $+X$ can be written as:

$$
\begin{aligned}
\frac{d \sigma^{j e t s}}{d E_{T} d \eta}= & \sum_{i} \iiint d x_{i} d Q^{2} d \ldots \\
& \cdot\left[d k_{\perp}^{2} x_{i} \mathcal{A}\left(x_{i}, k_{\perp}^{2}, \bar{q}\right)\right] \hat{\sigma}\left(x_{i}, k_{\perp}^{2}\right)
\end{aligned}
$$
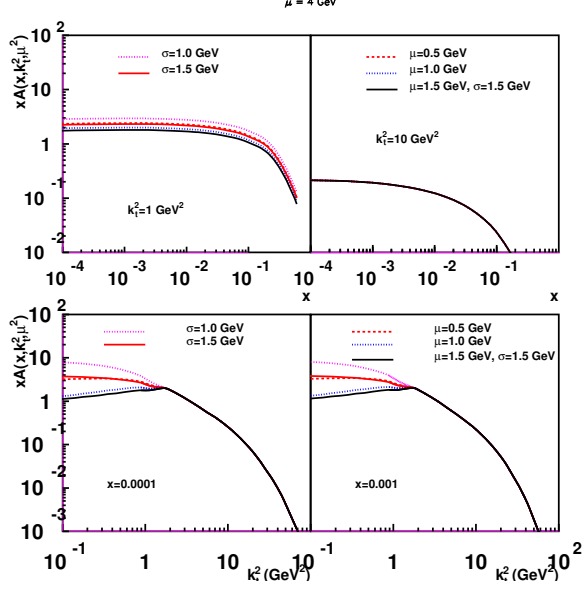

Figure 1: The unintegrated gluon distribution at a scale $\bar{q}=4 \mathrm{GeV}$ for different values of At high energies, the gluon density is dominating for many processes, therefore here only the gluon $\mathrm{uPDF}$ is considered. It has already been shown in [9], that the predic$\mu$ and $\sigma$ of the intrinsic $k_{\perp}$ distribution as a function of $x$ for fixed $k_{\perp}$ (top) and as a function of $k_{\perp}$ (bottom) for fixed $x$ tions of the total cross section as well as differential distributions for heavy quark production at HERA and the LHC agree well in general with those coming from fixed NLO calculations. However, the details depend crucially on a precise knowledge of the uPDF. Therefore precision fits to inclusive and exclusive measurements have to be performed to determine precisely the free parameters of the uPDF: the starting distribution function at a low scale $Q_{0} \sim 1 \mathrm{GeV}$ as well as parameters connected with $\alpha_{\mathrm{s}}$ and details of the splitting functions for the perturbative evolution.

An overview and discussion of uPDFs is given in [4-6]. In a previous paper [10] the uPDF was determined from a pQCD fit using the CCFM evolution equation [11-14] to the 
structure function $F_{2}$ and $F_{2}^{c}$ with acceptable $\chi^{2} / n d f$. However, the small $x$ behavior of the uPDF obtained from $F_{2}^{c}$ was very different compared to the one obtained from $F_{2}$.

Here also measurements of high $p_{t}$-dijet production in DIS at HERA [15-17] are investigated.

\section{The method}

The unintegrated gluon density is determined by a convolution of the non-perturbative starting distribution $\mathcal{A}_{0}(x)$ and the CCFM evolution denoted by $\tilde{\mathcal{A}}\left(x, k_{\perp}, \bar{q}\right)$ :

$$
x \mathcal{A}\left(x, k_{\perp}, \bar{q}\right)=\int d x^{\prime} \mathcal{A}_{0}\left(x^{\prime}, k_{\perp}\right) \cdot \frac{x}{x^{\prime}} \tilde{\mathcal{A}}\left(\frac{x}{x^{\prime}}, k_{\perp}, \bar{q}\right)
$$

In the perturbative evolution the gluon splitting function $P_{g g}$ including non-singular terms

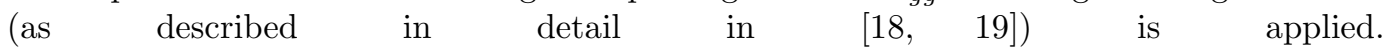

The distribution $\mathcal{A}_{0}$ is parameterized at the starting scale $Q_{0}$ by:

$$
\begin{aligned}
x \mathcal{A}_{0}\left(x, k_{\perp}\right)= & N x^{-B_{g}} \cdot(1-x)^{C_{g}}\left(1-D_{g} x\right) \\
& \cdot \exp \left[-\frac{\left(\mu-k_{\perp}\right)^{2}}{\sigma^{2}}\right]
\end{aligned}
$$

The parameters $N_{g}, B_{g}, C_{g}, D_{g}$ as well as $\mu, \sigma$ of $\mathcal{A}_{0}$ are free parameters which have to be constrained by measurements. It turns out, that $C_{g}, D_{g}$ are not sensitive to the data considered here, and are therefore fixed to $C_{g}=4$ and $D_{g}=0$. The other parameters are determined by a fit [20] to measurements such to minimize the $\chi^{2}$ defined by:

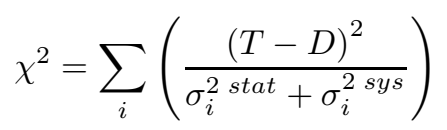

with $T$ being the theory value and $D$ the measurement with the corresponding statistical and systematic uncertainty.

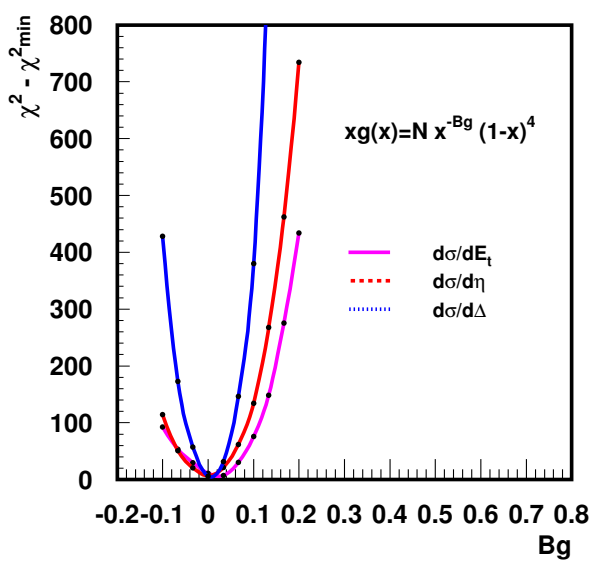

Figure 2: A scan in the parameter space of $B_{g}$ for $\frac{d^{3} \sigma}{d Q^{2} d x d E_{t}}, \frac{d^{3} \sigma}{d Q^{2} d x d \Delta}$ and $\frac{d^{3} \sigma}{d Q^{2} d x d \Delta \eta}$ as measured in [15].

\section{The intrinsic $k_{\perp}$ distribution}

The Gaussian form with $\mu=0$ and a width of $\sigma \sim 1.0 \mathrm{GeV}$ of the intrinsic $k_{\perp}$ distribution in eq.(1) is an assumption to parameterize our ignorance about the small $k_{\perp}$ behavior. In the saturation model of GBW [21] the uPDF vanishes for small $k_{\perp}$. Such a behavior can be mimicked by a Gaussian distribution with $\mu \sim Q_{0}$. The effect of choosing different $\mu$ is illustrated in Fig. 1. 


\section{Dijets in DIS}

The sensitivity of the shape in $x$ and the intrinsic $k_{\perp}$ was studied for dijets in DIS [15] in the kinematic range of $5<Q^{2}<100 \mathrm{GeV}^{2}, 10^{-4}<x<10^{-2}, 0.1<y<0.7$ and two jets with at least $E_{t}>5 \mathrm{GeV}$ in the range $-1<\eta<2.5$. The differential cross sections $\frac{d \sigma}{d E_{t}}, \frac{d \sigma}{d \Delta \eta}$, with $\Delta \eta$ being the rapidity difference between the highest $E_{t}$ jets are mainly sensitive to the $x$ dependence of the uPDF. The same is observed for the cross section $\frac{d \sigma}{d \Delta}$ with $E_{t}>E_{t \min }+\Delta$ and $E_{t \text { min }}=5 \mathrm{GeV}$. A scan over the parameter space of $B g$ is shown in Fig 2. With this choice of parameters the cross sections are well described, giving a reasonable $\chi^{2} / n d f$. In Tab. 1 the $\chi^{2} / n d f$ are given for different values of $B g$ and the mean $\mu$ of the intrinsic $k_{\perp}$ distribution.

\begin{tabular}{|l|c|c|c|c|}
\hline & & \multicolumn{3}{|c|}{$\chi^{2} / n d f$} \\
\hline $\mathrm{Bg}$ & $\mu[\mathrm{GeV}]$ & $\frac{d \sigma}{d E_{t}}$ & $\frac{d \sigma}{d \Delta \eta}$ & $\frac{d \sigma}{d \Delta}$ \\
\hline 0.025 & 1.5 & $68 / 37=1.8$ & $102 / 35=2.3$ & $267 / 89=3.0$ \\
\hline 0.25 & 1.5 & $95 / 37=2.5$ & $113 / 35=2.5$ & $306 / 89=3.4$ \\
\hline 0.025 & 0 & $63 / 37=1.7$ & $93 / 35=2.1$ & $284 / 89=3.2$ \\
\hline 0.25 & 0 & $99 / 37=2.7$ & $123 / 35=2.7$ & $345 / 89=3.9$ \\
\hline
\end{tabular}

From Tab. 1 it is seen, that a value of $B g=0.025$ is preferred, and that the sensitivity of these measurements to the intrinsic $k_{\perp}$ distribution is very small.

However, the cross

Table 1: Quality of the description of the different differential cross sections using $B g=0.025$ and $B g=0.25$ together with $\sigma=1.5 \mathrm{GeV}$.

section as a function of $\Delta \phi$, where $\Delta \phi$ is the difference in azimuthal angle between the two leading jets in the hadronic center-of-mass frame, is directly sensitive to the transverse momentum of the incoming parton, and thus a crucial test of the uPDF.

In Fig. 3 we show a comparison of the measurement of [17] with the prediction of CASCADE using the uPDF determined before. A reasonable description of the measurement is achieved. Table 2 shows the $\chi^{2} / n d f$ obtained for these data and also to the azimuthal correlations from [16]. It is interesting to observe, that $\frac{d \sigma}{d \Delta \phi}$ gives also

\begin{tabular}{|l|c|c|c|}
\hline & & \multicolumn{2}{|c|}{$\chi^{2} / n d f$} \\
\hline $\mathrm{Bg}$ & $\mu[\mathrm{GeV}]$ & $\frac{d \sigma}{d Q^{2} d \Delta \phi}(\mathrm{H} 1 \mathrm{prel})$ & $\frac{d \sigma}{d \Delta \phi}($ dijets ZEUS $)$ \\
\hline 0.025 & 1.5 & $163 / 29=5.6$ & $332 / 19=17.5$ \\
\hline 0.25 & 1.5 & $128 / 29=4.4$ & $234 / 19=12.3$ \\
\hline 0.025 & 0 & $200 / 29=6.9$ & $417 / 19=22.0$ \\
\hline 0.25 & 0 & $237 / 29=8.2$ & $338 / 19=17.8$ \\
\hline
\end{tabular}
access to $B g$, now with a preference to a much steeper initial gluon distribution. The measurement prefers a distribution which decreases for very small transverse momenta $k_{\perp}$. However it should be noted, that the form of the intrinsic $k_{\perp}$

Table 2: Quality of the description of $\frac{d \sigma}{d \Delta \phi}$ using $B g=0.025$ and $B g=0.25$ together with $\sigma=1.5 \mathrm{GeV}$ by H1 [16] and ZEUS [17]. distribution is not constrained.

\section{Conclusion}


The shape of the starting gluon distribution in $x$ and $k_{\perp}$ has been investigated with dijet events in DIS. Whereas the cross sections as a function of $E_{t}$ prefer a soft gluon distribution $(B g \sim 0.025)$ and show little sensitivity to the intrinsic $k_{\perp}$ distribution, the cross sections as a function of $\Delta \phi$ prefer a much steeper gluon $(B g \sim 0.25)$ and show a clear preference to a intrinsic $k_{\perp}$ distribution which decreases for small $k_{\perp}$. The different $x$-slope of the initial gluon distribution, as already observed in fits to $F_{2}$ and $F_{2}^{c}$, is also observed in di-jet cross section measurement. Further investigations are obviously needed.

\section{Acknowledgments}

Many thanks go to the the organizers of this
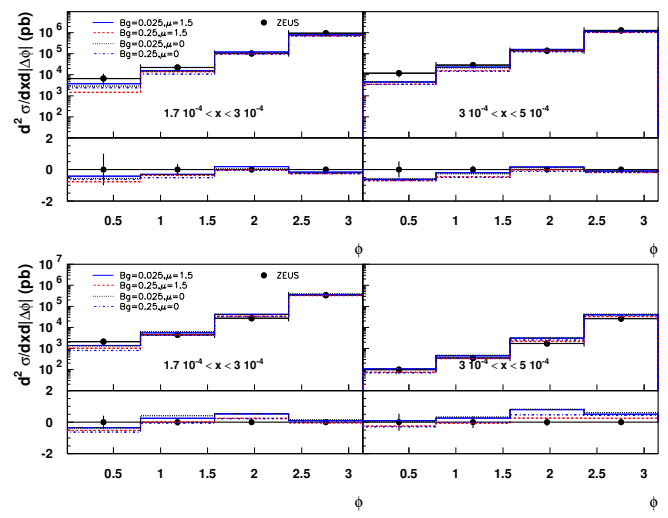

Figure 3: The cross section $\frac{d \sigma}{d \Delta \phi}$ as measured by [17] compared to predictions using CASCADE and the uPDF as in Tab. 2. The lower plots always show the ratio $R=\frac{\text { theory-data }}{\text { data }}$.

\section{References}

[1] Slides: http: //indico. cern. ch/contributionDisplay $\cdot$ py? contribId=54\&sessionId=6\& conf Id=9499

[2] H. Jung, Phys. Rev. D 65, 034015 (2002). [hep-ph/0110034]

[3] H. Jung, Mod. Phys. Lett. A19, 1 (2004). [hep-ph/0311249]

[4] Small X Collaboration; B. Andersson et al., Eur. Phys. J. C 25, 77 (2002). [hep-ph/0204115]

[5] Small X Collaboration, J. R. Andersen et al., Eur. Phys. J. C35, 67 (2004). [hep-ph/0312333]

[6] Small X Collaboration, J. R. Andersen et al. (2006). [hep-ph/0604189]

[7] H. Jung. DIS 2004, Strbské Pleso, Slovakia, [hep-ph/0411287]

[8] J. Collins and H. Jung, Need for fully unintegrated parton densities, 2005. [hep-ph/0508280]

[9] S. Alekhin et al., Hera and the LHC - a workshop on the implications of HERA for LHC physics: Proceedings Part $A$ and B, 2005. [hep-ph/0601012,hep-ph/0601013], J. Baines et al. (2006). [hep-ph/0601164]

[10] H. Jung, A. V. Kotikov, A. V. Lipatov and N. P. Zotov, [hep-ph/0611093].

[11] M. Ciafaloni, Nucl. Phys. B 296, 49 (1988)

[12] S. Catani, F. Fiorani, and G. Marchesini, Phys. Lett. B 234, 339 (1990)

[13] S. Catani, F. Fiorani, and G. Marchesini, Nucl. Phys. B 336, 18 (1990)

[14] G. Marchesini, Nucl. Phys. B 445, 49 (1995)

[15] A. Aktas et al. [H1 Collaboration], Eur. Phys. J. C 33 (2004) 477 [hep-ex/0310019].

[16] M. Hansson [H1 Collaboration], "Decorrelation Of Dijets At Low X And Q**2," Prepared for 14th International Workshop on Deep Inelastic Scattering (DIS 2006), Tsukuba, Japan, 20-24 Apr 2006

[17] S. Chekanov et al. [ZEUS Collaboration], [hep-ex/0705.1931] .

[18] H. Jung, Acta Phys. Polon. B33, 2995 (2002). [hep-ph/0207239]

[19] M. Hansson and H. Jung. DIS 2003, St. Petersburg, Russia, [hep-ph/0309009]

[20] F. James and M. Roos, Comput. Phys. Commun. 10, 343 (1975)

[21] K. Golec-Biernat and M. Wusthoff, Phys. Rev. D 60 (1999) 114023 [hep-ph/9903358]. 